\title{
An Efficient Blind Detection Algorithm of Median Filtered Image
}

\author{
Yongzhen $\mathrm{Ke}^{1^{*}}$, Fan Qin ${ }^{2}$, Weidong $\mathrm{Min}^{3}$ and Qiang Zhang ${ }^{4}$ \\ ${ }^{1,3,4}$ School of Computer Science and Software Engineering, Tianjin Polytechnic \\ University, China \\ ${ }^{2}$ Department of Logistics Management, Nankai University, China \\ fannq@163.com keyongzhen@tjpu.edu.cn,minweidong@tjpu.edu.cn, \\ 442786301@qq.com
}

\begin{abstract}
Due to the significant advances of digital photography and the availability of many powerful photo editing tools, it becomes easier to create forgery images by non-professional users. Median filtering that is usually applied to erase the forensically significant fingerprints has recently received increased attention. In this paper, we present an effective blind forensic algorithm to detect the median filtering manipulation. First, the median filtered residual $(M F R)$ is generated by computing the difference between a testing image and a median filtered version of itself. Then, three feature sets including histogram, autocorrelation and gradient are extracted from the median filter residual. Last, those features are fetched into support vector machine (SVM) for training and classification. Our experimental results demonstrate that our proposed forensic method achieves not only better detection rate but also lower computational complexity compared with other existing median filtering detection methods. Our proposed forensic method also can locate local median filtering of image effectively.
\end{abstract}

Keywords: Image forensic, Median filtering, Autocorrelation, Histogram, Gradient, SVM

\section{Introduction}

With the advent of the Internet and low-price digital cameras, as well as powerful image editing software, digital images have found wide applications in news media, military, and law enforcement. Meanwhile, the authenticity of digital images can no longer be taken for granted. In recent years, many image forgery detection techniques have been proposed, especially passive or blind forensic methods which do not require any additional information besides the image itself with undetermined authenticity. By extracting features that capture the underlying statistics of an image, tampering can be blindly distinguished from authentic data [1]. Existing image forensic works involve the detection of median filtering (MF) [2-4], resampling [5-6], JPEG compression [7], blur [8-9] and so on. Median filtering is a nonlinear operation that has the useful property of preserving edges within an image. It is commonly used to perform image denoising, remove outlying pixel values, and smooth regions of an image. Because of this, forgers may use median filtering to make their image forgeries appear more perceptually realistic. Therefore, blind detection of non-linear median filtering becomes especially important.

In this paper, we provide an efficient blind detection algorithm for reliable MF detection in digital images. Based on the properties observed in an image's median filter residual (MFR), which is defined as the difference between an image in question and a median filtered version of itself [4], three new feature sets are constructed for MF detection. Those features are fed into a support vector machine (SVM) for classification. The trained classifier is applied to 
discriminate a median filtered image from a non-altered image. Compared with other existing median filtering forensic techniques [4, 10-11], the proposed method yields better or at least comparable performance and has ability of detecting local median filtering in an image.

This paper is organized as follows. Section 2 presents the related works. The proposed methods are described in detail in Section 3. Experimental procedure and results are discussed in Section 4. Finally, conclusions and future work are drawn in Section 5.

\section{Related Work}

Blind detection method for image median filtering can be divided into two categories: one is based on pixel value difference of image, and another is using the difference between an image in question and a median filtered version of itself.

Kirchner and Fridrich [3] believed that the streaking artifact was a specific characteristic of median filtered images, and proposed a detector that operated based on the statistics of pixel value differences of a median filtered image and an unaltered image. The detector first divided the image into blocks, the pixel difference histogram in each of the block was calculated. Next, the ratio ${ }^{\rho}$ of the number of pixel differences whose value was zero to the number of pixel differences whose value was one was calculated for each block. A weighting function was applied to the ${ }^{\rho}$ value of each block to avoid statistical distortion in saturated regions and a final measure ${ }^{\wedge} \rho$ of the strength of median filtering fingerprints was obtained. Gang Cao et al. [10] proposed a MF detection scheme by analyzing the probability that the first order pixel difference of an image will be zero in textured regions. Furthermore, they demonstrated that their technique can distinguish median filtering from rescaling, Gaussian filtering, and average filtering. While they were able to demonstrate that this technique can very effectively detect median filtering in uncompressed images, its performance degraded significantly in JPEG compressed images. Haidong Yuan [2] proposed detecting median filtering by measuring the relationships among pixels within a $3 \times 3$ window. A set of $44 \mathrm{D}$ median filtering features (MFF) including the distribution of the block median pixel value and the distribution of the number distinct gray levels within a window were extracted. The experimental results indicated that MFF-based approach can achieve comparable or better performance than SPAM-based method [3] on both high and moderate quality JPEG and detecting median filtering on small image windows. However, as with the SPAM-based detector, the performance of MFF-based detector degraded as the JPEG quality factor decreased or the size of the analyzed image shrank. Thus, there was a need to develop more reliable schemes to detect median filtering in the case of strong JPEG compression and low resolution, which was more desirable in practical applications. Chenglong Chen and Jiangqun Ni [12] proposed a median filtering detection method by calculating the edge based prediction matrix (EBPM) of different kinds of image edges and obtained 72 dimensions of prediction coefficients to differentiate median filtering. They used a prediction model of the pixel values in image regions with different gradients and captured statistical relationships between nearby pixels to perform median filtering detection. In their recent work [1, 13], they exploited cumulative distribution function of first-order and second-order image difference as fingerprints to construct the global probability feature set (GPF). They also used the local correlations between different adjacent image difference pairs to construct the local correlation feature set (LCF). They finally used GPF and LCF to construct a new feature set GLF of 56 dimensions. Their method achieved good performance for low resolution and JPEG compression.

Xiangui Kang et al. [4, 14] introduced a robust median filtering detection scheme by analyzing the statistical properties of the median filter residual (MFR). Median filtering was 
first applied on a test image, and the difference between the initial image and the filtered output image was called the median filtered residual (MFR). The MFR was used as the forensic fingerprint. To capture the statistical properties of the MFR, an autoregressive (AR) model of the MFR was calculated and the AR coefficients were used by a support vector machine (SVM) for classification. Their experimental results shown that their proposed forensic technique can achieve important performance gains, particularly at low false-positive rates, with a very small dimension of features. Fangling Shi [11] proposed a JPEG images median filter detection algorithm. The median filtering with $3 \times 3$ was first applied to a test JPEG image, and the test JPEG image was calibrated by setting up a threshold. Then the difference between the calibrated image and filtered image was calculated. Finally twodimensional features for detecting median filtering including total number of pixel point in the difference image where its grey value was zero and the mean value of the difference image were used to detect whether JPEG image was applied median filtering operation or not. Junyu Xu and Yuting Su [15] proposed a novel algorithm for detecting smoothing filtering in digital images based on the frequency residual. The suspected image was re-filtered with a gaussian low-pass filter, and the difference between the initial image and the re-filtered image in Fourier domain was called the frequency residual. Then, the frequency residual was projected into the Radon space with an adaptation of Radon transform. The obtained data were modeled as Fourier series and the model parameters were adopted as features for filtering detection. Their experimental results shown that the proposed algorithm can not only detect three typical smoothing spatial filters, including Gaussian filter, average filter, and median filter, but also can predict parameters of these filters.

\section{The Proposed Algorithm}

\subsection{Analysis of Median Filtering}

The median filter operates by replacing a pixel value with the median value of the pixels in a small window surrounding it. The most commonly used median filter windows are squares of size $3 \times 3$ and $5 \times 5$ pixels. Median filtering is performed window by window with the windows overlapping each other [4]. Given an $M \times N$ grayscale image $X_{i, j}$ with $(i, j) \in(1, \ldots, M) \times(1, \ldots, N)$, a 2 -D median filter is defined as

$$
\begin{aligned}
& y(i, j)=\text { median }\left\{x(i+h, j+v) \mid(h, v) \in\left(-\frac{w-1}{2}, \ldots, 0, \ldots, \frac{w-1}{2}\right)\right. \\
& (i, j) \in(1, \ldots, M) \times(1, \ldots, N)
\end{aligned}
$$

where $x(i, j)$ is the pixel value at point $(i, j), y(i, j)$ is the output of the median filter at image coordinates $(i, j), \operatorname{median}\{\bullet\}$ is the median operator and $w$ is the 2-D filter window centered at image coordinates $(i, j)$.

The median filter is a well-known non-linear filter based on order statistics. Due to its nonlinearity, a theoretical analysis of the general relation between input and output distributions of the median filter is very cumbersome. Hence, the analysis of median filtering has largely been confined to more specific features of interest [3]. A median filter not only smooths noise and preserves edges in images, but also tends to produce regions of constant or nearly constant value, which produce streaks (linear patches) or amorphous blotches. Bovik [16] analyzed the phenomenon of streaking/blotching in median-filtered signals quantitatively, and computed the probability that the median values stemming from overlapping windows were equal. It is noted that neighboring pixels in median filtered images are correlated to some extent because they originate from overlapping windows of the 
original signal. With the inherent nature of the median filter, it is expected that pixels in median filtered images are correlated to their neighbors in a specific way [13].

\subsection{Feature Sets for Median Filter Detection}

As mentioned in Section 2, the pixel value difference of image has been taken as the forensic trace [2-3, 10, 12-13]. However, the pixel value difference contains largely the local content of an image, such as edge and texture information. The edge and texture interfere with the median filtering detector and thus deteriorate the performance. Figure 1(b) shows an image along with its first order pixel difference taken in the horizontal direction of an image (Figure 1a). We can clearly see in figure 1(b) that the first order difference contains a great deal of the image's edge and texture content. This edge information and the block artifacts may affect the conditional first order difference distributions used by SPAM to detect median filtering. In order to suppress both image content and block artifacts, the difference between a median filtered version of an image and the image itself (called as the median filtered residual [14]) is a good choice. Figure 1(c) shows that the median filtered residual contains less edge and texture information than the first order pixel difference.

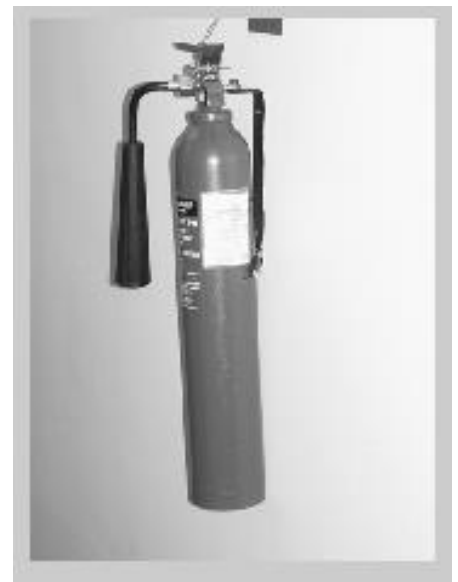

(a) An Image

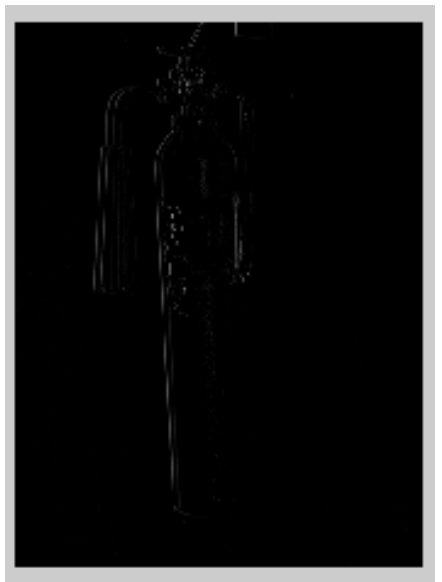

(b) Its First Order Difference

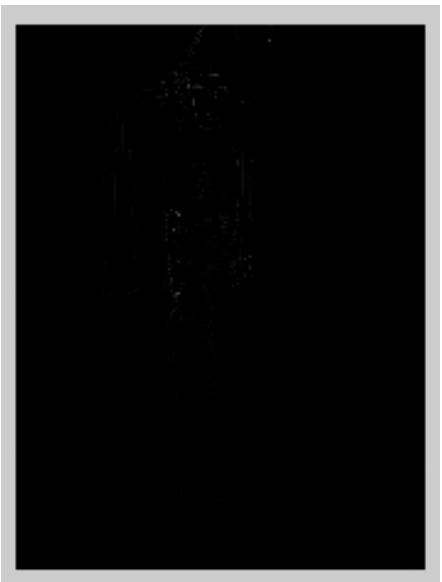

(c) Its Median Filter Residual

Figure 1. Example Showing

Based on the median filtered residual, three new features for detecting median filtering are observed in this paper. First, similar to [3], the pixel histogram is also applied to the median filtered residual for first feature. Figure 2 shows the histogram of the median filtered residual on original image and its median filtered image. From the Figure 2, we can clearly see that the histogram curves of median filtered image (MF) are substantially different from the ones of original image. 


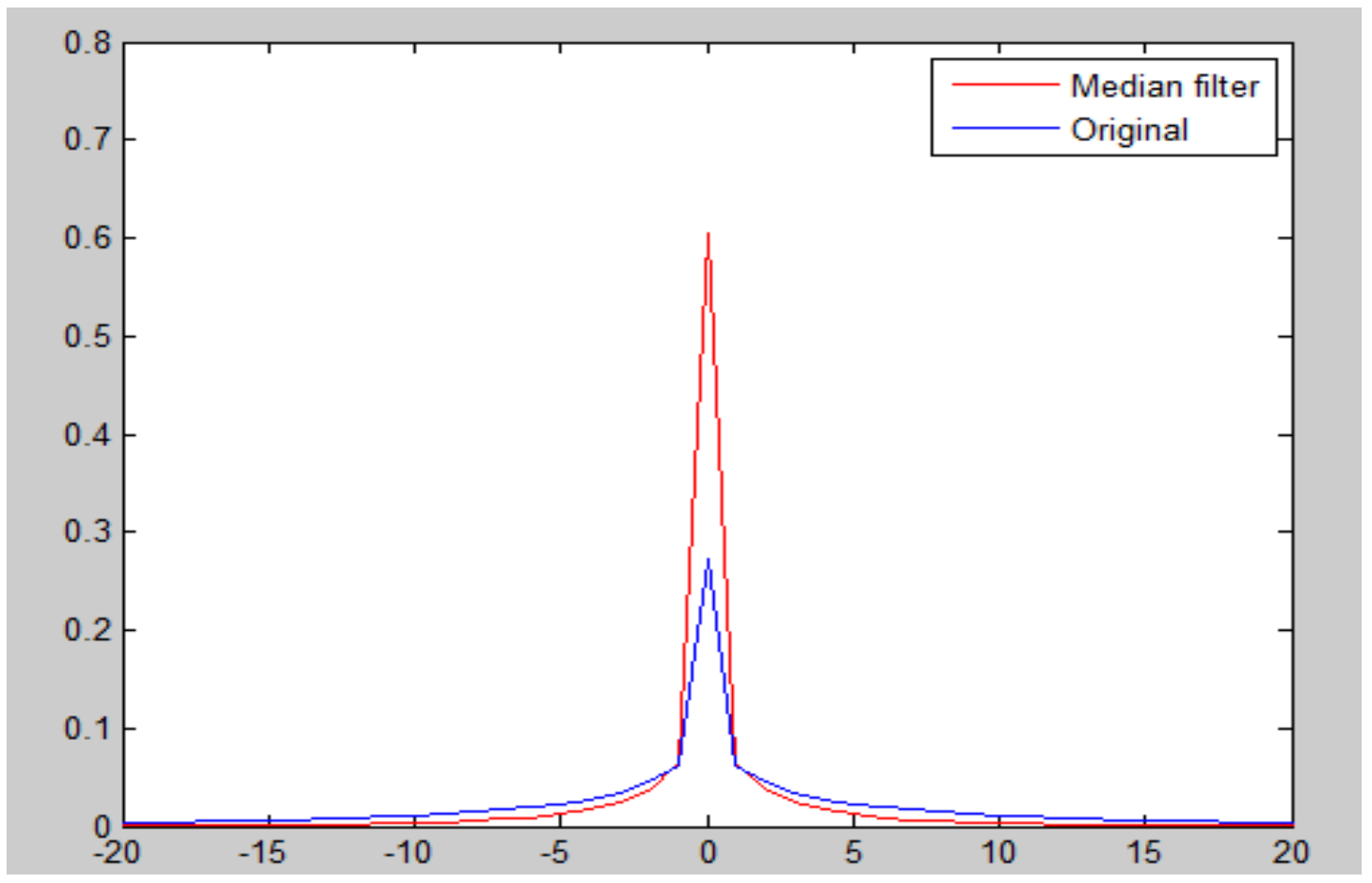

Figure 2. Histogram of the Median Filtered Residual on Original Image and its Median Filtered Image

Because the overlapped window filtering introduces correlation among the pixels of MFR, we model the MFR using a simple autocorrelation function instead of autoregressive (AR) model [4]. Figure 3 also suggests a clear distinction between the autocorrelation coefficients of the median filtered residual of median filtered image and original image.

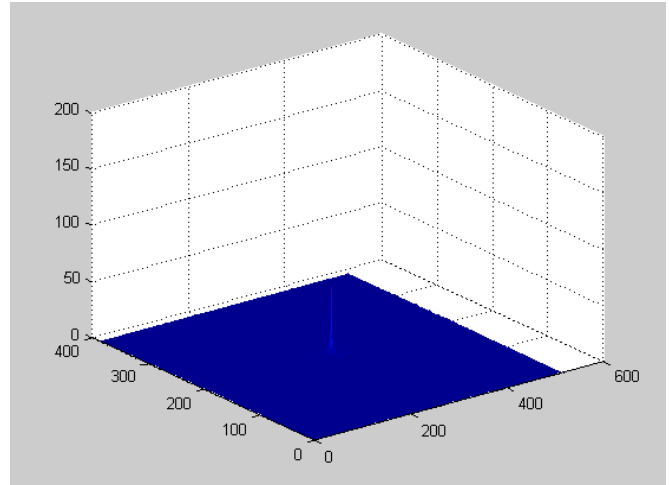

(a)Original Non-Filtered Image

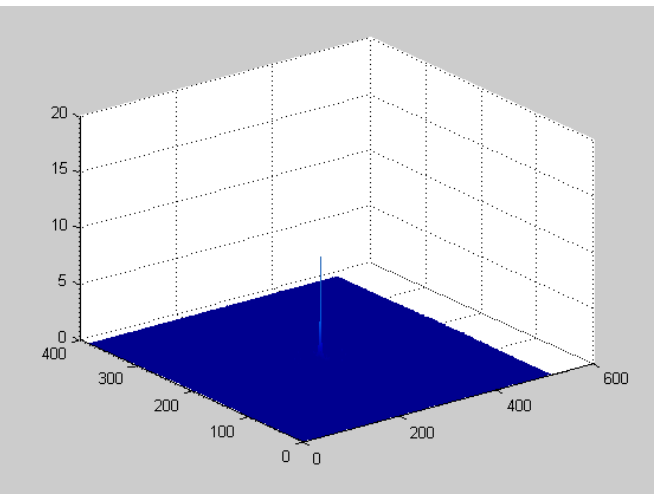

(b) Median Filtered Image

Figure 3. The Autocorrelation Coefficients of the Median Filtered Residual

Similar effects are also observed in the gradient of the median filtered residual as shown in Figure 4. It is observed that the gradient of the median filtered residual can be used as fingerprint for MF. 


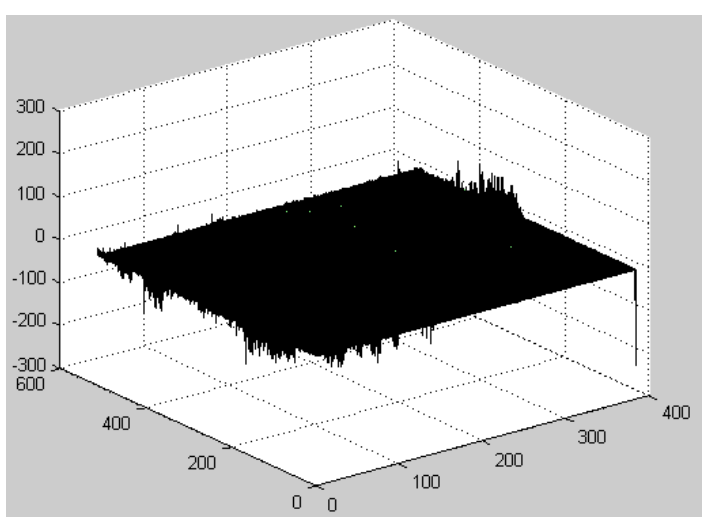

(a)Original Non-Filtered Image

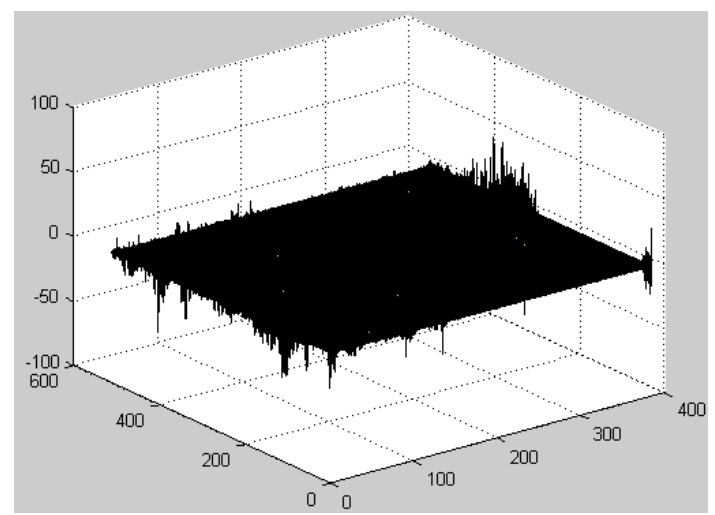

(b) Median Filtered Image

\section{Figure 4. The Gradient of the Median Filtered Rresidual}

\subsection{A Blind Detection Algorithm of Median Filtered Image}

According to the analysis in Section 3.2, an efficient blind detection algorithm of median filtered image is proposed as following:

(1)Obtain median filtered residual image. For all images with size of $\mathrm{M} \times \mathrm{N}$, the median filtered residual $d_{(x, y)}$ is

$$
d(x, y)=I^{\prime}(x, y)-I(x, y), \quad(x, y) \in(1, \cdots, M) \times(1, \cdots, N)
$$

where $I(x, y)$ is the testing image and $I^{\prime}(x, y)$ is its median filtered image.

(2) Construct the first set of features based on the histogram feature of median filtered residual image. We compute the normalized histogram $H(n)$ :

$$
H(n)=\frac{\#\{(x, y): d(x, y)=n\}}{N},-255 \leq n \leq 255
$$

where $N$ is the total number of pixels in filtered residual image $d(x, y)$, and \# denotes the cardinal number of a set.

(3) Construct the second set of features based on the autocorrelation function of median filtered residual image. Let's consider a $2 \mathrm{~d}$ signal $\mathrm{X}$, its auto-correlation function $R_{x}(u, v)$ is related to the power spectral density $S_{x}(a, b)$ via the Wiener-Khinchine theorem [17] :

$$
S_{x}(a, b)=\int_{-\infty}^{+\infty} \int_{-\infty} R_{x}(a, b) e^{-2 \pi i(a u+b v)} d u d v=F\left[R_{x}(a, b)\right]
$$

On the other hand, the power spectral density is the square modulus of the Fourier transform of the $2 \mathrm{~d}$ signal $\mathrm{X}$ :

$$
S_{x}=|F(X)|^{2}=F(X) \cdot F(\bar{X})
$$

with $F(X)$, the Fourier transform, and $F(\bar{X})$ the conjugate transform .

$$
F(X)=Y(a, b)=\sum_{u=1}^{N} \sum_{v=1}^{M} X(u, v) \cdot \exp \left(\frac{-2 \pi i u a}{N}+\frac{-2 \pi i v b}{M}\right)
$$




$$
F(\bar{X})=Y(\bar{a}, b)=\sum_{u=1}^{N} \sum_{v=1}^{M} X(u, v) \cdot \exp \left(\frac{2 \pi i u a}{N}+\frac{2 \pi i v b}{M}\right)
$$

From the equations (4) and (5), the auto-correlation function of the Signal $\mathrm{X}$ is given by :

$$
R(X)=F^{-1}[F(X) \cdot F(\bar{X})]
$$

Two-dimensional features including peak value and variance of autocorrelation coefficient $R(X)$ are extracted.

(4) Construct the third set of features based on the gradient of median filtered residual image. The gradient of an image is given by the formula:

$$
\begin{aligned}
& G x(x, y)=I(x+1, y)-I(x, y) \\
& G y(x, y)=I(x, y+1)-I(x, y)
\end{aligned}
$$

where $G x$ and $G y$ are the gradient in the $\mathrm{x}$ and $\mathrm{y}$ direction respectively. We compose a set of 8 dimension gradient features including the mean, variance, skewness, and kurtosis of $G x$ and $G y$.

(5) Three feature sets are fed into a support vector machine (SVM) for classification between median filtered images and unaltered images. We employ a LSSVM with RBF kernel as the classifier. We used the "grid-search" method to find the optimal parameters $\sigma$ and ${ }^{\gamma}$ of RBF kernel.

The proposed method is illustrated in Figure 5.

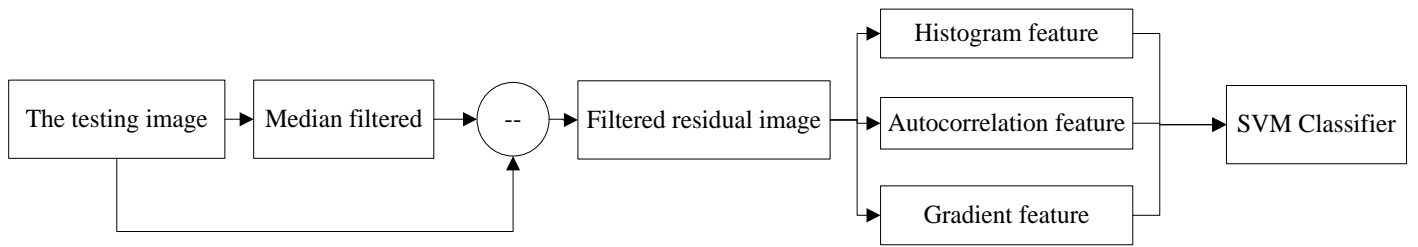

Figure 5. The Proposed Blind Detection Algorithm of Median Filtered Image

\section{Results and Discussion}

To evaluate the effectiveness of our proposed median filtering method and to compare its performance with other existing median filtering detection techniques, the popular image dataset UCID [18] is introduced for test. The use of databases and the procedure of experiment are inspired by [4] and [10]. The UCID dataset consists of 1338 uncompressed TIFF images on a variety of topics including natural scenes and man-made objects, both indoors and outdoors. Note that all images are color images and converted into grayscale in the standard manner.

\subsection{The Classification Performance}

In the first case, we compared our proposed method with [4] in the same experiments manner. All color images were first converted to gray scale images before further processing. Median filtered images were generated by performing $3 \times 3$ median filtering and $5 \times 5$ median filtering on the unaltered gray-scale images. Each unaltered and median filtered image was then saved in both its uncompressed state and JPEG compressed state using a variety of quality factors ranging between 90 and 30. Table 1 shows the results of our proposed method and [4], where some results refereed from table 1 in [4], in which the minimal average 
decision error of each technique under the assumption of equal priors and equal costs is defined as

$$
P_{e}=\min \left(\frac{P_{f p}+1-P_{t p}}{2}\right)
$$

where ${ }_{P_{f p}}$ and ${ }_{P_{t p}}$ denote the false positive (FP) and true positive rates (TP), respectively.

Table 1. Classification Results Compared with [4]

\begin{tabular}{|l|l|l|l|l|l|}
\hline \multicolumn{2}{|l|}{} & MF 3×3 & MF 5 5 & \\
\hline Quality factor & & The Proposed Method & {$[4]$} & The Proposed Method & {$[4]$} \\
\hline JPEG 90 & $\mathrm{P}_{\mathrm{e}}(\%)$ & 0.64 & 0.5 & 2.59 & 0.5 \\
\hline & $\mathrm{P}_{\mathrm{tp}}(\%)$ & 99.76 & 99.5 & 99.53 & 99.7 \\
\hline & & & & \\
\hline JPEG 70 & $\mathrm{P}_{\mathrm{e}}(\%)$ & 1.06 & 1.3 & 1.88 & 1.0 \\
\hline & $\mathrm{P}_{\mathrm{tp}}(\%)$ & 99.53 & 97.5 & 99.9 & 98.8 \\
\hline JPEG 50 & $\mathrm{P}_{\mathrm{e}}(\%)$ & 0.59 & 2.2 & 1.53 & 2.1 \\
\hline & $\mathrm{P}_{\mathrm{tp}}(\%)$ & 99.9 & 93.5 & 99.06 & 95.8 \\
\hline
\end{tabular}

It is observed from table 1 that our proposed method achieves nearly perfect classification performance and has better performance than [4] in most instance but MF $5 \times 5$ using quality factor of 90 , where the $\mathrm{P}_{\mathrm{tp}}$ is 99.53 , and is lower than 99.7 of [4].

In the second case, all color images were first converted to gray scale images and then saved in JPEG compressed state using a variety of quality factors ranging between 95 and 75 . Median filtered images were generated by performing $3 \times 3$ median filtering and $5 \times 5$ median filtering on the compressed gray-scale images. We selected 100 images of compressed grayscale images and its Median filtered images as SVM training dataset, others as testing dataset. The procedure of experiment is the same as [11]. Table 2 shows the results of our proposed method and [10, 11], where some results refereed from Table 4.1 and 4.2 in [11]. The results in Table 2 indicate that our scheme performs better performance than $[10,11]$.

Table 2. Classification Results Compared with $[10,11]$

\begin{tabular}{|c|c|c|c|c|c|c|c|c|c|}
\hline & \multicolumn{3}{|l|}{ MF $3 \times 3$} & \multicolumn{3}{|l|}{ MF $5 \times 5$} & \multicolumn{3}{|l|}{ MF 7×7 } \\
\hline $\begin{array}{l}\text { quality } \\
\text { factor } \\
\text { (QF) }\end{array}$ & $\begin{array}{l}\text { The } \\
\text { Proposed } \\
\text { Method }\end{array}$ & [11] & [10] & $\begin{array}{l}\text { The } \\
\text { Proposed } \\
\text { Method }\end{array}$ & [11] & [10] & $\begin{array}{l}\text { The } \\
\text { Proposed } \\
\text { Method }\end{array}$ & [11] & [10] \\
\hline JPEG 95 & 97.0 & 89.0 & 66.5 & 96.0 & 93.75 & 73.5 & 98.0 & 96.5 & 80.5 \\
\hline JPEG 90 & 96.0 & 84.75 & 63.75 & 95.0 & 92.0 & 71.25 & 98.0 & 96.0 & 77.0 \\
\hline JPEG 85 & 97.0 & 83.25 & 62.25 & 95.0 & 91.75 & 69.25 & 98.0 & 96.0 & 73.75 \\
\hline JPEG 80 & 98.0 & 82.5 & 62.0 & 92.0 & 91.25 & 67.75 & 98.0 & 96.0 & 73.75 \\
\hline JPEG 75 & 95.0 & 81.75 & 71.75 & 92.0 & 91.25 & 65.25 & 95.0 & 95.75 & 72.5 \\
\hline JPEG 70 & 97.0 & 81.0 & 60.25 & 92.0 & 90.75 & 63.75 & 99.0 & 90.75 & 70.5 \\
\hline
\end{tabular}

Last, similar to second case, we test our proposed method on performing 3 x 3 average filtering, $5 \times 5$ average filtering and $7 \times 7$ average filtering on the compressed gray-scale images. The results shown in Table 3 also indicate that our proposed method performs good performance on average filtering. 
Table 3. Classification Results of Average Filtering

\begin{tabular}{|l|l|l|l|}
\hline quality factor (QF) & $3 \times 3$ average filtering & $5 \times 5$ average filtering & $7 \times 7$ average filtering \\
\hline JPEG 95 & 1.0 & 99.75 & 1.0 \\
\hline JPEG 90 & 99.5 & 99.75 & 1.0 \\
\hline JPEG 85 & 99.75 & 99.75 & 1.0 \\
\hline JPEG 80 & 99.25 & 99.5 & 1.0 \\
\hline JPEG 75 & 99.0 & 99.75 & 1.0 \\
\hline JPEG 70 & 99.5 & 99.75 & 1.0 \\
\hline
\end{tabular}

\subsection{Detecting Local Median Filtering in an Image}

In order to test the ability of our proposed method for detecting local median filtering forgeries when a portion of a median filtered image is inserted into a non-median filtered image, or a portion of an image is performed median filter operation, two experiments are finished in this paper. First, we give an example of a cut-and-paste image forgery in which the pasted region has undergone median filtering before it is inserted into an unaltered image in Figure 6. Figure 6(a) and 6(b) show two original JPEG compressed images using a quality factor of 90 from UCID database. The forged image shown in Figure 6(c) was created by inserting part of the $3 \times 3$ median-filtered version of Figure 6(a) into the unaltered image in Figure 6(b). In order to detect the forgery, the forged image was first segmented into nonoverlapping image blocks of $R \times R$ pixels ( $\mathrm{R}=32,64$, and 128), then each block was tested to detect whether each image block was applied median filtering operation or not. Figure 6(d), (e), (f) show the results of detections on non-overlapping image blocks of $32 \times 32,64 \times 64$, and $128 \times 128$ pixels using our proposed method, where detected median filtered blocks are marked by green boxes. Figure $6(\mathrm{~g})$ shows the results of detections on overlapping image blocks of $64 \times 64$ pixels. The evaluation results demonstrate that overlapping image blocks with size of $64 \times 64$ achieve the best result. This example shows that our method achieves good performance in the cut-and-paste forgery detection.

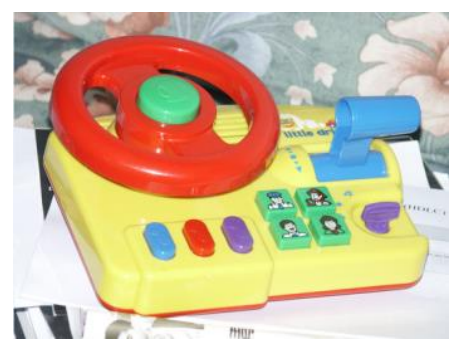

(a) The Original Image

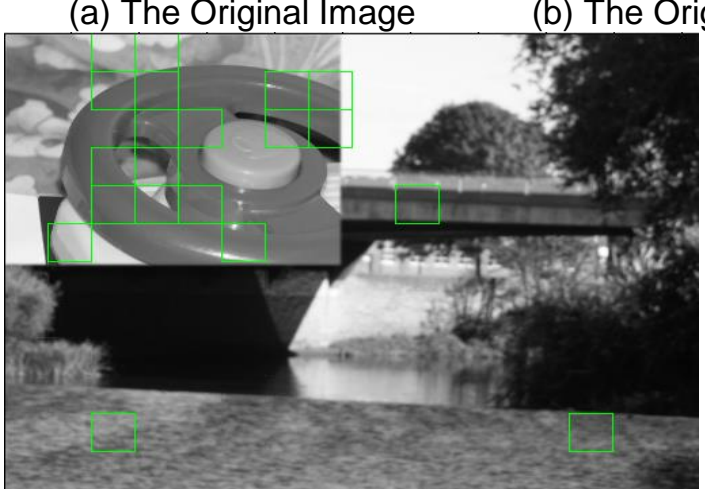

(d) The result of non-overlapping image blocks of $32 \times 32$

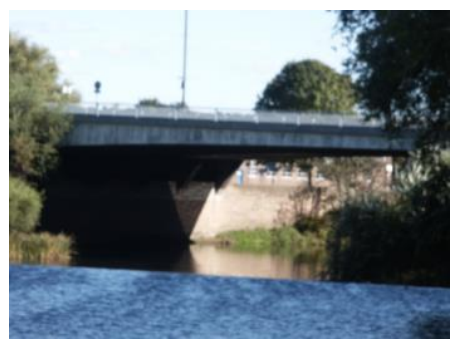

(b) The Original Image

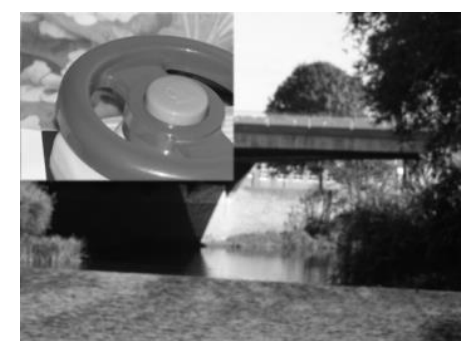

(c) The Forged Image

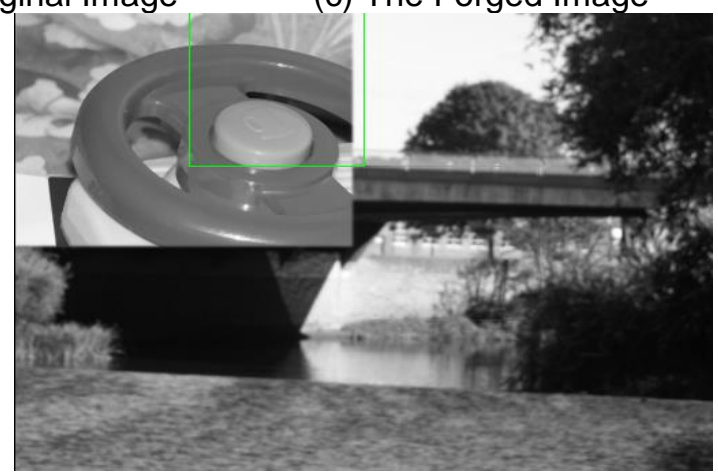

(e) The result of non-overlapping image blocks of $128 \times 128$ 


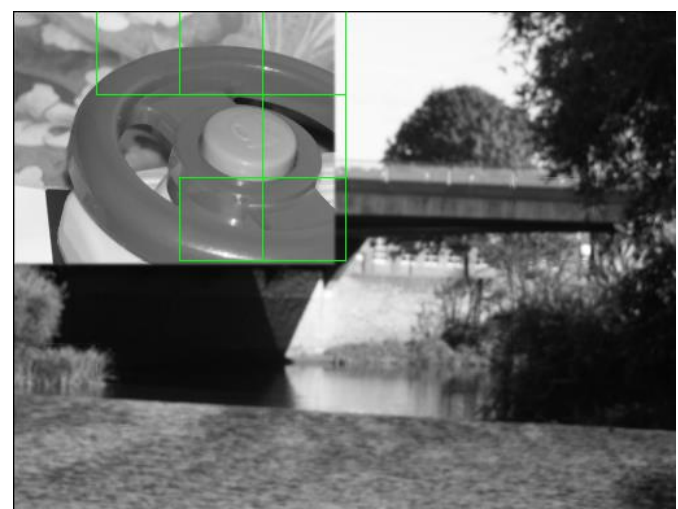

(f) The result of non-overlapping image blocks of $64 \times 64$

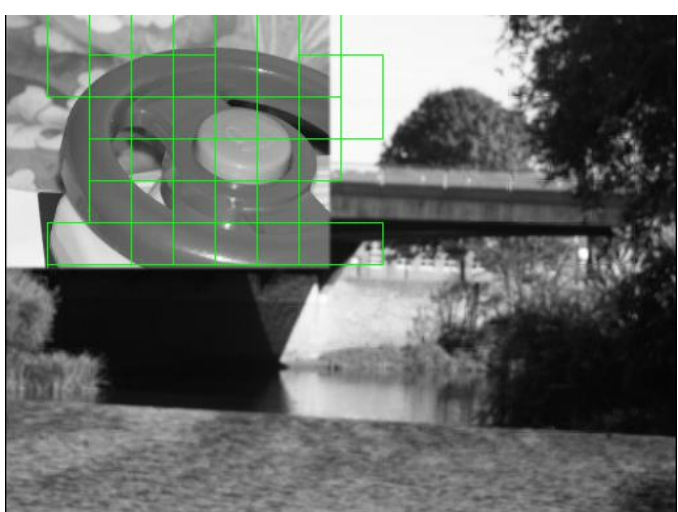

(g) The result of overlapping image blocks of $64 \times 64$

Figure 6. Cut and Paste Forgery Detection Example

Second, another example is shown in Figure 7. Figure 7(a) shows the original images. The local median filtered image shown in Figure 7 (b) was created by performing the $3 \times 3$ median filter operation on the center block of image. As shown in Figure 7(c) our proposed method can reliably locate the local median filtered in the compressed forgery.

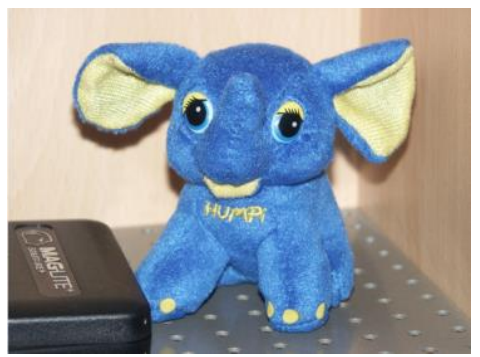

(a) The original image

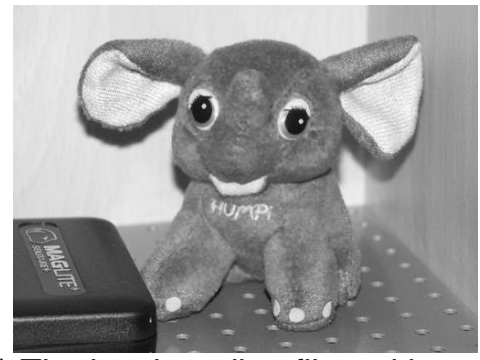

(b) The local median filtered image

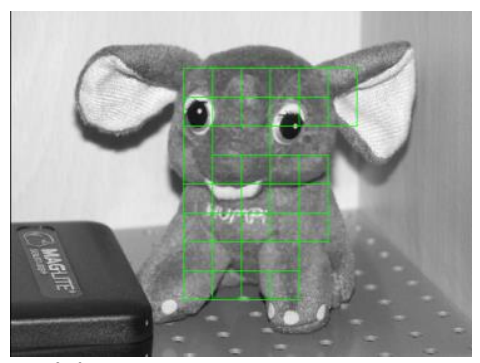

(c) The detection result

Figure 7. The Detection Result of Detecting Local Median Filtering

\section{Conclusions}

In this paper, we present an effective forensic algorithm to detect the median filtering manipulation, which is usually applied to erase the forensically significant fingerprints. We extract three new detection features including histogram, autocorrelation, and gradient from the difference between a median filtered version of an image and the image itself. Last, those features are fetched into SVM for training and classification. A series of experiments are designed to test MF detection performance. The experimental results show that our method has better detection rate with a very small dimension of features compared with other method and also can effectively detect local median filtering in an image. In the future work, we are interested in combining the current detection method with other methods to increase the detection performance and the range of the forensic applications.

\section{Acknowledgements}

This research was partially supported by the Natural Science Foundation of Tianjin, China (Grant No. 13JCYBJC15500). 


\section{References}

[1] C. Chen, J. Ni and J. Huang, "Blind Detection of Median Filtering in Digital Images: A Difference Domain Based Approach", IEEE Transactions on Image Processing, vol. 22, no.12, (2013), pp. 4699-4710.

[2] H. Yuan, "Blind Forensics of Median Filtering in Digital Images", IEEE Transactions on Information Forensics and Security, vol. 6, no. 4, (2011), pp. 1335-1345

[3] K. Matthias and F. Jessica, "On Detection of Median Filtering in Digital Images", Proceedings of the IS\&T/SPIE Electronic Imaging, (2010), pp. 754110--754110.

[4] X. Kang, M. C. Stamm, A. Peng and K. J. Ray Liu, "Robust Median Filtering Forensics Using an Autoregressive Model", IEEE Transactions on Information Forensics and Security, vol. 8, no. 9, (2013), pp. 1456-1468.

[5] P. Alin C and F. Hany, "Exposing Digital Forgeries by Detecting Traces of Resampling", IEEE Transactions on Signal Processing, vol. 53, no. 2 II, (2005), pp. 758-767.

[6] Kirchner and Matthias, "On the Detectability of Local Resampling in Digital Images", Proceedings of the SPIE of Security, Forensics, Steganography, and Watermarking of Multimedia Contents X, (2008), pp. 68190F-68190F-11

[7] N. Ramesh, R. De Queiroz, F. Zhigang, D. Sanjeeb and B. Richard G, "JPEG Compression History Estimation for Color Images", IEEE Transactions on Image Processing, vol. 15, no. 6, (2006), pp. 1365-1378

[8] X. Wang, B. Xuan and S. Peng, "Digital Image Forgery Detection Based on the Consistency of Defocus Blur", Proceedings of the Fourth International Conference on Intelligent Information Hiding and Multimedia Signal Processing (IIH-MSP), (2008), pp. 192-195

[9] P. Kakar, S. Natarajan and W. Ser, "Detecting Digital Image Forgeries through Inconsistent Motion Blur, Proceedings of the IEEE International Conference on Multimedia and Expo (ICME), (2010), pp. 486-491

[10] G. Cao, Y. Zhao, R. Ni, L. Yu and H. Tian, "Forensic Detection of Median Filtering in Digital Images", Proceedings of the 2010 IEEE International Conference on Multimedia and Expo (ICME), (2010), pp. 89-94

[11] F. Shi, "Research on Double Compression JPEG Image Detection and Median Filtering Detection", Master Dissertation, (2012), Northwestern University (In Chinese).

[12] C. Chen and J. Ni, "Median Filtering Detection using Edge Based Prediction Matrix", Proceedings of the International Workshop on Digital Forensics and Watermarking, (2012), pp. 361--375.

[13] C. Chen, J. Ni, R. Huang and J. Huang, "Blind Median Filtering Detection using Statistics in Difference Domain", Proceedings of the 14th International Conference Information Hiding, (2013), pp. 1--15.

[14] X. Kang, M. C. Stamm, A. Peng and K. J. Ray Liu, "Robust Median Filtering Forensics Based on the Autoregressive Model of Median Filtered Residual", Proceedings of the Signal \& Information Processing Association Annual Summit and Conference, (2012), pp. 1-9.

[15] J. Xu and Y. Su, "Smoothing Filtering Detection for Digital Image Forensics", Journal of Electronics \& Information Technology, vol. 35, no. 10, (2013), pp. 2287-2293. (In Chinese)

[16] B. Alanconrad, "Streaking in Median Filtered Images", IEEE Transactions on Acoustics, Speech and Signal Processing, vol. 35, no. 4, (1987), pp. 493--503.

[17] Wiener-Khinchin theorem, http://en.wikipedia.org/wiki/Wiener\%E2\%80\%93Khinchin_theorem\#cite_note-0

[18] S. Gerald and S. Michal, "UCID: an Uncompressed Color Image Database", Proceedings of the SPIE, Storage and Retrieval Methods and Application for Multimedia, (2004), pp. 472--480

\section{Authors}

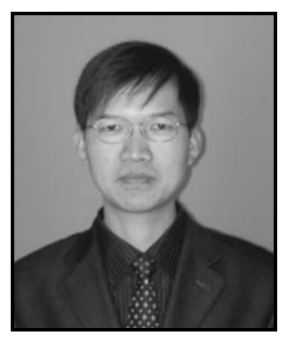

Yongzhen Ke, he obtained his BE of computer application at Tianjin Polytechnic University in China in 1997, and received his ME and $\mathrm{PhD}$ of computer application at Tianjin University in China in 2000 and 2008, respectively, on the research subjects of information security, image processing and data visualization. From 1997 to the present he worked in Tianjin Polytechnic University, China. Now he is an associate professor at School of Computer Science \& Software Engineering, Tianjin Polytechnic University, China. His current research interests include image processing, digital image forensic, network security and application of IoT. 


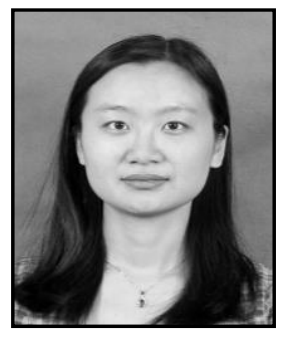

Fan Qin, she obtained her bachelor degree of economics at Nankai University in China in 2000, and received her master degree and $\mathrm{PhD}$ of management at Nankai University in China in 2005 and 2009, respectively, on the research subjects of information management, data mining. From 2009 to the present she worked in Nankai University, China. Now she is a lecture at department of logistics management, Nankai University, China. Her current research interests include information system management, image recognition, e-commerce and logistics management.

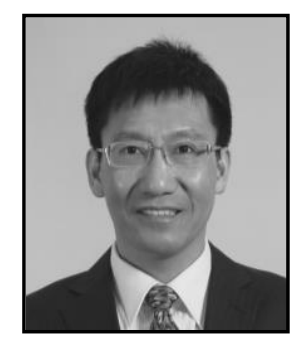

Weidong Min, he obtained his $\mathrm{BE}, \mathrm{ME}$ and $\mathrm{PhD}$ of computer application at Tsinghua University in China in 1989, 1991 and 1995, respectively, on the research subjects of computer graphics, image processing and computer aided geometric design. He was an assistant professor of Tsinghua University from 1994 to 1995. From 1995 to 1997 he was a postdoctoral researcher at University of Alberta, Canada. From 1998 to 2011 he worked as a senior research project manager at Corel, March Networks and other companies in Canada. Now he is an associate professor at School of Computer Science \& Software Engineering, Tianjin Polytechnic University, China. His current research interests include computer graphics, image and video processing, software engineering, distributed system, network resources management.

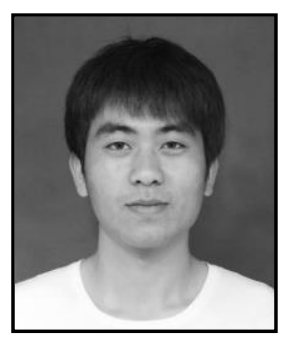

Qiang Zhang, he obtained her BE of computer application at Tianjin Polytechnic University in China in 2011, and she is a postgraduate at Tianjin University in China now, on the research subjects of image processing and digital image forensic. 